\title{
DESENLACES DEL MANEJO NO QUIRÚRGICO POSTERIOR A NEOADYUVANCIA DEL CÁNCER LOCALMENTE AVANZADO DE RECTO
}

\section{PILAR ADRIANA TORRES MESA}

Trabajo de investigación y Tesis de grado

Tutores temáticos:

Dr. Ricardo Oliveros Wilches - Jefe Clinica de Gastroenterología

Dr. Jorge A. Mesa - Patólogo Oncológico

Dra. Natalia Olaya - Patóloga Oncológica

Tutor metodológico:

Dr. Ricardo Sánchez - Investigación Clínica

INSTITUTO NACIONAL DE CANCEROLOGIA

UNIVERSIDAD MILITAR MUEVA GRANADA

CIRUGIA GASTROINTESTINAL ONCOLOGICA Y ENDOSCOPIA DIGESTIVA

BOGOTA DC, COLOMBIA

2014 


\section{DESENLACES DEL MANEJO NO QUIRÚRGICO POSTERIOR A NEOADYUVANCIA DEL CÁNCER LOCALMENTE AVANZADO DE RECTO}

Correspondencia: Pilar Adriana Torres Mesa, Avenida 1 No. 9-85, Clínica de Gastroenterología, Instituto Nacional de Cancerología, Tel: 3341111 , Fax: 3341111, Cel: 3124317583, Email: piadriana78@gmail.com 


\section{CONTENIDO}

INTRODUCCIÓN

1. PACIENTES Y MÉTODOS

$\begin{array}{ll}\text { 1.1. Selección de pacientes } & 10\end{array}$

1.2. Análisis patológico y de marcadores 12

1.3. Análisis estadístico 13

2. RESULTADOS 14

$\begin{array}{ll}\text { 3. DISCUSIÓN } & 20\end{array}$

4. CONCLUSIONES 28

$\begin{array}{lr}\text { 5. BIBLIOGRAFÍA } & 29\end{array}$ 


\section{LISTA DE TABLAS}

Tabla 1. Determinación del estado TNM, antes y después de la neoadyuvancia 


\section{LISTA DE GRÁFICOS}

Gráfica 1. Función de supervivencia libre de enfermedad

Gráfica 2. Función de supervivencia global

Gráfica 3. Algoritmo de manejo del cáncer de recto

Gráfica 4. Seguimiento recomendado para los pacientes con respuesta clínica completa post neoadyuvancia sin manejo quirúrgico radical 


\section{RESUMEN}

Objetivos: Describir características clínicas, histopatológicas y desenlaces, de pacientes con cáncer localmente avanzado de recto y respuesta clínica completa posterior a la neoadyuvancia, sin manejo quirúrgico.

Métodos: Estudio de cohorte retrospectiva de pacientes con cáncer de recto estadios II y III, llevados a quimiorradiación, en seguimiento clínico. Se evaluó supervivencia libre de enfermedad, global y tasa de recaída. Se describen características biológicas (Kras, Ki67, p53) y morfológicas del tumor (grado, invasión linfovascular y perineural). Resultados: Entre enero de 2003 y junio de 2013, 19 pacientes con cáncer localmente avanzado de recto y respuesta clínica completa postneoadyuvancia, no aceptaron el tratamiento quirúrgico radical. Con mediana de seguimiento de 21 meses (4-92 meses), se presentaron recaídas del $21 \%$ el primer año, $36 \%$ a los 3 años y $42 \%$ a los 5 años (total: 8 pacientes). Se presentó recaída local en $50 \%$ de casos, regional en $50 \%$ y no hubo recaída sistémica. La tasa estimada de recaída local fué 2,3 recaídas por 100 pacientes/mes (IC 95\%:1,21-4,5) y de recaída regional 1,3 recaídas por 100 pacientes/mes (IC 95\%:0.5-3,1). No se estableció relación, entre la expresión de factores biológicos del tumor primario y los desenlaces.

Conclusiones: Se ratifica la indicación del tratamiento quirúrgico radical, posterior a la neoadyuvancia, en todos los pacientes con cáncer localmente avanzado de recto. Las bajas tasas de recaída local y regional de nuestra serie, sugieren la posibilidad de resección local u observación, en casos seleccionados. La individualización y deseo del paciente, debe orientar la toma de decisiones.

Palabras clave: Cáncer de recto; Terapia neoadyuvante; Respuesta clínica completa; Manejo no quirúrgico; Marcadores tumorales. 


\section{INTRODUCCIÓN}

El cáncer colorectal es el cuarto tipo de cáncer más frecuentemente diagnosticado y la segunda causa de muerte en EEUU en el $2013^{1}$. En Europa ocupa el segundo lugar y a nivel mundial el tercero ${ }^{2}$. En Colombia ocupa el quinto lugar en la mortalidad general por cáncer, con una tasa de 5.3 por 100.000 habitantes y un incremento anual promedio del 1.9 a $2.2 \%{ }^{3-4}$.

La estadificación clínica del cáncer de recto, se realiza con la clasificación TNM (TNMc), de la $7^{\text {th }}$ edición de la American Joint Comission on Cancer ${ }^{2,4-6}$. Para ello, se requiere un examen clínico completo, toma de antígeno carcinoembrionario (ACE), colonoscopia y biopsia para confirmación histológica del tumor, tomografía computada (TC) tórax, abdomen y pelvis, resonancia nuclear magnética (RNM) de pelvis y/o ultrasonografía rectal (UES). Con ello se logra una precisión diagnóstica de la estadificación clínica entre el 83 y $96 \%^{5-10}$. Si hay dudas sobre el compromiso sistémico, intolerancia a los medios de contraste o alguna contraindicación para el uso del mismo, debe considerarse el uso de PET-CT, aunque no este rutinariamente indicado ${ }^{11}$.

El tratamiento estándar de los tumores localmente avanzados de recto, es la neoadyuvancia con quimiorradiación ${ }^{12-15}$. Consiste en quimioterapia con base en fluoropirimidinas ${ }^{16-17}$, que se administra con dosis biológicas efectivas de radioterapia concomitante $^{14-15,18}$. La efectividad se demuestra con el mayor tiempo libre de enfermedad, disminución del volumen tumoral, de la estadificación inicial y de la tasa de recaída local y regional, aunque no se ha demostrado aumento de la supervivencia global $^{12-13,19}$.

Al finalizar la neoadyuvancia se reestadifica el tumor con la clasificación TNMy y en todos los pacientes se plantea la programación de resección quirúrgica del tumor, 6 a 
10 semanas posterior a la finalización del tratamiento ${ }^{20-21}$, si se descarta progresión o irresecabilidad de la enfermedad.

Entre el 15 al 30\% de los pacientes presentan respuesta clínica completa posterior a la neoadyuvancia ${ }^{22}$. Significa que durante el examen clínico o endoscópico en el lecho tumoral se evidencia: un área de aclaramiento de la mucosa, telangiectasias, mínima pérdida de la flexibilidad de la pared rectal, una cicatríz, un nódulo apenas perceptible, o no hay nada ${ }^{23-24}$. El reporte de las biopsias es negativo para malignidad, con un grado de regresión histológica completa ${ }^{26}$; existe ausencia del compromiso ganglionar y mesorrectal visualizado en RNM de abdomen y pelvis o ultrasonografía rectal y se descarta el compromiso metastásico en las imágenes diagnósticas citadas o tomografía por emisión de positrones (PET-CT) $)^{27-30}$.

Existen pacientes con enfermedades asociadas, alto riesgo quirúrgico o que no aceptaron la cirugía dentro de su esquema de manejo, quienes empezaron un plan de seguimiento clínico, imagenológico y endoscópico frecuente por presentar respuesta clínica completa posterior a la quimiorradiación. Se ha reportado en ellos, aceptables tasas de supervivencia libre de enfermedad, variables tasas de recaída y buena calidad de vida, en contraste con los pacientes que fueron llevados a procedimientos quirúrgicos radicales y ostomías ${ }^{31-38}$. La primera cirujano en exponer el tema fue la Dra Angelita Habr Gama, quien con su grupo de colaboradores, han planteado la opción de "watch and see" en pacientes seleccionados, que consiste en observar y valorar la evolución de esos pacientes con respuesta clínica completa, con miras al diagnóstico y manejo precoz de una recaída o reportar sus desenlaces ${ }^{19,23-24,35-38}$. En los pacientes que presentan recaída de la enfermedad, se puede ofrecer la realización de una resección local ${ }^{39-43}$, nuevos esquemas de quimioterapia ${ }^{44}$, manejo quirúrgico derivativo, paliativo o radical, según el caso particular.

Eso ha motivado la investigación sobre las características histopatológicas y biológicas 
del tumor (nivel de antígeno carcinoembrionario (ACE) ${ }^{45-46}$, tipo y grado histológico, estado mutacional del Kras, invasión linfovascular y perineural ${ }^{47}$, inestabilidad de microsatélites del DNA, p57, Ki67) ${ }^{48-53}$. También se han determinado alteraciones genómicas, de factores de crecimiento endotelial y vasculares ${ }^{54-57}$. Todos ellos parecen actuar como factores predictores de respuesta y pronósticos ${ }^{58-60}$.

El objetivo de este estudio es describir los desenlaces de los pacientes con cáncer localmente avanzado de recto, respuesta clínica completa postneoadyuvancia, sin tratamiento quirúrgico posterior por decisión del paciente, valorados en nuestra institución y con seguimiento regular. Las variables histopatológicas y factores biológicos del tumor se determinaron en cada caso. 


\section{PACIENTES Y MÉTODOS}

\subsection{Selección de pacientes}

Se realizó una revisión retrospectiva de los datos clínicos e histopatológicos recogidos prospectivamente. La muestra es de pacientes con confirmación histológica de adenocarcinoma de recto, con borde distal dentro de los $12 \mathrm{cms}$ del margen anal y que cumplían las siguientes características:

-Estadío clínico II (T3-4, N0) y III (cualquier T, N1-2) según la clasificación TNM.

-Recibieron tratamiento con quimioterapia (5-Fluorouracilo), concomitantemente con radioterapia (40-55 Gy) con intención neoadyuvante por 4 a 6 semanas, en el Instituto Nacional de Cancerología o extrainstitucional, con el mismo protocolo empleado.

-En la reestadificación clínica postneoadyuvancia se determina que presentaron respuesta clínica completa yTNM estadío 0 -I.

-No se realizó manejo quirúrgico oncológico, por decisión del paciente ó la concomitancia de enfermedades asociadas.

-Posibilidad de seguimiento en la Clínica de Cirugía Gastrointestinal Oncológica y Endoscopia Digestiva del Instituto Nacional de Cancerología, con disponibilidad de registros clínicos de su evolución.

-Disponibilidad de tejido tumoral antes y después de la neoadyuvancia, para la revisión del material histopatológico por el equipo de Patología.

La estadificación preoperatoria se realizó con el examen clínico y determinación del estado funcional (Escala de Karnosfsky), análisis sanguíneos (química sanguínea, perfil nutricional, hemograma y marcadores tumorales), colonoscopia, toma de biopsias de la lesión, tomografía de abdomen, pelvis, tórax y RNM abdominopélvica. Antes del año 
2011 no hay reportes de RNM en ningún paciente, debido a limitaciones económicas y técnicas para su realización. La estadificación postneoadyuvancia se realizó 2 a 8 semanas posterior a su finalización, con los mismos elementos diagnósticos descritos previamente. Se determinó una respuesta clínica completa, si al examen clínico o endoscópico no había evidencia del tumor o existían estigmas de su presencia (cicatriz palpable menor de $2 \mathrm{~cm}$, sin ulceración, no adherida a planos profundos, sin estenosis). Se tomó biopsia de la cicatriz o del sitio donde estaba previamente el tumor y se confirmó que era negativa para malignidad. La tomografía o RNM de abdomen y pelvis mostraban ausencia de progresión de la enfermedad y descartaba el compromiso mesorrectal o ganglionar regional.

A todos los pacientes se les informó desde la primera valoración y en los seguimientos, sobre la necesidad de realizar un procedimiento quirúrgico radical oncológico, como parte del manejo integral de su patología. Este dependía de la respuesta a la neoadyuvancia y la localización del tumor en el recto medio o inferior y debía realizarse 6 a 10 semanas posterior a la finalización de la quimiorradiación. Sin embargo, todos los pacientes de esta serie, se negaron a entrar en el proceso de planeación y realización del manejo quirúrgico.

Se planteó para ellos, la realización de un seguimiento clínico, endoscópico (rectosigmoidoscopia o colonoscopia con biopsias de la cicatriz), imagenológico (tomografía o RNM de abdomen y pelvis) y análisis sanguíneos (hemograma, química sanguínea, marcadores tumorales), cada 3 meses por los primeros 3 años y posteriormente cada 6 meses hasta completar los 5 años, después del cual se continuaría el seguimiento anual.

Todos los pacientes fueron suficientemente informados sobre el alto riesgo de recaída y que de presentarse, debía reestadificarse y considerarse el manejo oncológico integral, que podía incluir la necesidad de un procedimiento quirúrgico con o sin 
ostomía, estrategia de tratamiento entendida, aceptada y consignada en la historia clínica del paciente.

Se considera recaída, a la presencia de enfermedad dentro de los 5 años de seguimiento regular. Los tipos de recaída son: local (presencia de masa, estenosis, ulceración o rigidez de la pared rectal, en el sitio donde estaba localizado previamente el tumor, con biopsias positivas o no para malignidad); regional (engrosamiento de la pared rectal o pérdida del plano de clivaje con estructuras vecinas, compromiso mesorrectal o ganglionar regional evidenciado por tomografía ó RNM de abdomen y pelvis.); a distancia (compromiso peritoneal, hepático, pulmonar o de varios de ellos) o concomitancia de ellas.

\subsection{Análisis patológico y de marcadores}

Todos los pacientes tenían disponible tejido previo y posterior a la neoadyuvancia, embebido en parafina, lográndose la confirmación de adenocarcinoma de recto por el grupo de Patología de la institución. Se determinaron la presencia o no de invasión linfovascular y perineural, grado y tipo histológico. El p53 se considera de expresión positiva, si presenta una tinción mayor del $5 \%$ en las células tumorales. Por técnicas de inmunohistoquímica se determina el marcador de proliferación Ki67, el cual se establece como de baja proliferación si es menor del $20 \%$, media entre el 20 y $50 \%$ y alta si es mayor del 50\%. Se determinó el estado mutacional Kras en los tejidos disponibles. El grado de regresión histológica se evaluó en las biopsias postneoadyuvancia. Para ello se utilizó la clasificación de Rodel modificada, la que se interpreta como completa (ausencia de células viables en la muestra), moderada (presencia de pocas células residuales en menos del $50 \%$ de la muestra), mínima (células residuales en mas del $50 \%$ de la muestra o fibrosis importante), ausente (sin

regresión histológica) y no determinada (material insuficiente o mal estado de la 
muestra para determinarla).

\subsection{Análisis estadístico}

El análisis estadístico fue desarrollado con el programa SPSS versión 20. Se utilizaron métodos convencionales de estadística descriptiva, (uso de medianas junto con sus correspondientes medidas de dispersión o de frecuencia y proporciones) para la caracterización de las variables relacionadas con las características generales del paciente, que corresponden al momento del diagnóstico y después de la neoadyuvancia.

Se establece la supervivencia libre de enfermedad, como el periodo en meses, desde la fecha de finalización de la neoadyuvancia hasta la fecha de la recaída si se presentó o el último control en la institución.

La supervivencia global corresponde al tiempo en meses desde la finalización de la neoadyuvancia hasta la muerte del paciente ó último control en la institución.

Tanto el tiempo hasta la muerte como el tiempo hasta la recaída se analizaron con métodos de supervivencia (cálculo de la función de supervivencia de Kaplan-Meier). Adicionalmente se calculó la tasa de recaída, para lo cual se tomo como denominador el tiempo en meses de seguimiento. Se discrimina como tasa de recaída local, regional, a distancia y global y se estiman junto con sus intervalos de confianza del $95 \%$.

Se establece la mediana de intervalo de seguimiento postneoadyuvancia, como el tiempo entre la fecha de finalización de la neoadyuvancia y la fecha de la recaída. También la mediana de tiempo de seguimiento post-recaída, como el tiempo entre la fecha de la recaída y la del último control clínico o muerte del paciente.

El diseño y realización del estudio fue aprobado por el comité de ética del Instituto Nacional de Cancerología. 


\section{RESULTADOS}

Entre enero de 2003 y junio de 2013, fueron valorados en el Instituto Nacional de Cancerología, en la Clínica de Cirugía Gastrointestinal Oncológica y Endoscopia Digestiva, 1370 pacientes con cáncer de recto. De ellos, 480 casos (35\%) fueron considerados tumores localmente avanzados y recibieron neoadyuvancia con quimiorradiación, según estadísticas institucionales. Sin embargo, no fue posible establecer en la serie global, el número de pacientes que presentaron respuesta clínica completa y fueron llevados a manejo quirúrgico radical.

Se identificaron 19 pacientes que cumplían los requisitos de inclusión y son la muestra de este estudio. Ellos corresponden a menos del $1 \%$ de la población, diagnosticada con cáncer rectal en ese periodo de tiempo y al $13 \%$ de los pacientes considerados con tumores localmente avanzados. Las razones de no aceptar el manejo quirúrgico oncológico, fueron la realización de una ostomía definitiva en el $47 \%$ (9 pacientes), temor al procedimiento en el $42 \%$ (8 pacientes) y riesgo quirúrgico alto por las enfermedades asociadas en el $37 \%$ ( 7 pacientes), existiendo concomitancia de las razones en 5 casos.

Las características demográficas del grupo son una mediana de edad de 65 años (rango 47-86 años), 9 mujeres (47 \%) y 10 hombres (53\%). La mediana de distancia del tumor al margen anal, fue $4 \mathrm{cms}$ (rango $1-10 \mathrm{cms}$ ). El estado de la clasificación TNM clínica del tumor y postneoadyuvancia se muestra en la tabla 1.

El $100 \%$ de los casos fueron adenocarcinoma de recto tipo clásico. Se identificaron 5 casos (26\%) de tumores bien diferenciados, 12 casos (63\%) de moderadamente diferenciados y $2(11 \%)$ tumores mal diferenciados. No se observó la presencia de infiltración perineural en las muestras. En 4 casos $(21 \%)$ se confirmó la invasión 
linfovascular.

Tabla 1. Determinación del estado TNM, antes y después de la neoadyuvancia

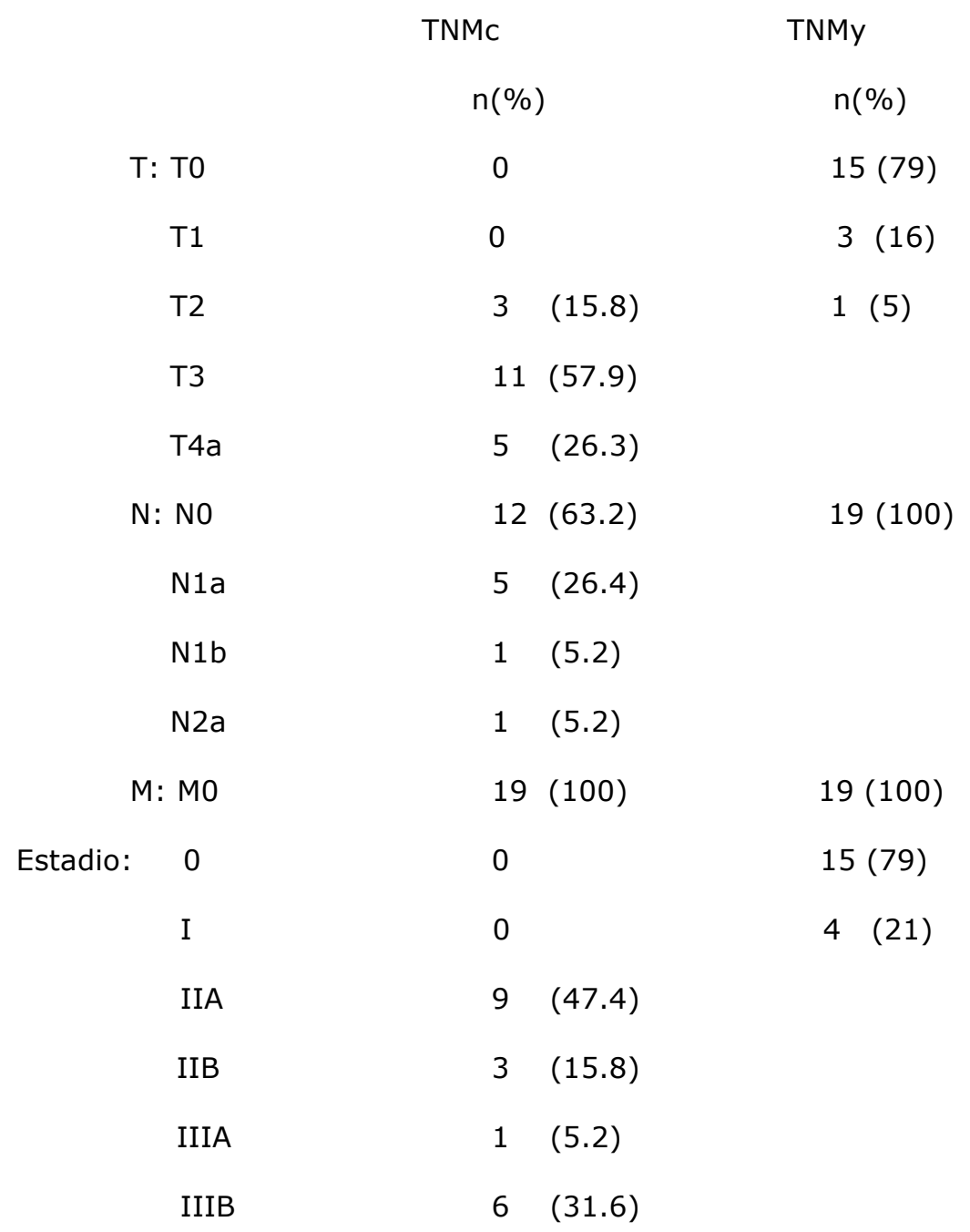

T: Tamaño del tumor, N: Compromiso ganglionar regional, M: Metástasis a distancia

La determinación de factores biológicos no fue posible en todos los especímenes de muestra. Las características histopatológicas de cada caso, se describen en la tabla 2. La mediana de seguimiento de la serie fue de 21 meses (Rango 4 a 92 meses). Las frecuencias de recaídas fueron: 4 pacientes (21\%) durante el primer año; 7 casos (36 \%) a los 3 años y 8 pacientes (42\%) a los 5 años.

La recaída local ocurrió en 4 casos (50 \%), regional en 4 casos (50 \%) y no se 15 
presentaron casos de recaída a distancia.

Tabla 2. Características del tumor de la biopsia inicial y postneoadyuvancia

\begin{tabular}{|c|c|c|}
\hline \multicolumn{2}{|c|}{ Características del tumor } & $\mathrm{n}=(\%)$ \\
\hline \multirow[t]{3}{*}{ Grado histológico: } & Bien & $5(26.3)$ \\
\hline & Moderado & $12(63.2)$ \\
\hline & Mal & $2(10.5)$ \\
\hline \multirow[t]{2}{*}{ Invasión perineural: } & Ausente & $13(68)$ \\
\hline & No determinada & $6 \quad(32)$ \\
\hline \multirow[t]{3}{*}{ Inv linfovascular: } & Presente & $4 \quad(21)$ \\
\hline & Ausente & $9(47.4)$ \\
\hline & No determinada & $6(31.6)$ \\
\hline \multicolumn{3}{|l|}{ Marcadores biológicos } \\
\hline \multirow[t]{3}{*}{ K ras: } & Mutado & $2(10.5)$ \\
\hline & No mutado & $2(10.5)$ \\
\hline & Sin dato & $15(79)$ \\
\hline \multirow[t]{3}{*}{ p53: } & Positivo & $2(10.5)$ \\
\hline & Negativo & $7 \quad(37)$ \\
\hline & Sin dato & $10(52.5)$ \\
\hline \multirow[t]{4}{*}{ Ki 67: } & Baja $(<20 \%)$ & $2(10.5)$ \\
\hline & Media (2 a $20 \%$ ) & $5 \quad(26.5)$ \\
\hline & Alta $(>$ a $20 \%)$ & $2(10.5)$ \\
\hline & Sin dato & $10(52.5)$ \\
\hline
\end{tabular}

Regresión tumoral postneoadyuvancia:

$\begin{array}{lll}\text { Completa } & 15(79) \\ \text { Moderada } & 3 & (15.8) \\ \text { Sin dato } & 1 & (5.2)\end{array}$

Se estima una tasa de recaída local de 2,3 recaídas por 100 pacientes/mes (IC $95 \%$ : 
1,21 a 4,5 recaídas) y una tasa de recaída regional de 1,3 recaídas por 100 pacientes/mes (IC $95 \%$ : 0.5 a 3,1 recaídas). El diagnóstico de la recaída se determinó en un $87,5 \%$ de los casos, por endoscopia. En todos los casos se tomó biopsia de la nueva lesión, la cual confirmó la reaparición tumoral en el 50 \% de los casos.

La mediana de intervalo de seguimiento postneoadyuvancia, hasta el diagnóstico de la recaída fue de 7 meses (rango 3 a 33 meses).

El manejo de la recaída fue quirúrgico en el $100 \%$ de los casos y se realizó durante las primeras 4 semanas, posteriores a su documentación. Sin embargo, en 2 pacientes se evidenció irresecabilidad de la lesión y se realizó ostomía derivativa, con el correspondiente manejo oncológico posterior.

Después del manejo integral del caso, la mediana de seguimiento post-recaída fué de 14,5 meses (rango 3 a 43 meses).

La presencia de la mutación Kras, p53 en las células tumorales y Ki67 en los pacientes con recaída, fue muy diversa y no se pudo encontrar significacia de su relación con el desenlace.

Sin embargo, tres pacientes (75\%) de los que presentaron invasión linfovascular, presentaron recaída antes de los dos años de seguimiento. Fueron recaídas locoregionales en 2 pacientes (50 \%) y local en un caso (25\%). La determinación de factores biológicos en estos tres eventos, no generó una constante que estuviera relacionada con el desenlace.

Uno de los pacientes presentó nuevamente enfermedad a los 74 meses. Se consideró como segundo primario, por que se presentó después de los 5 años del seguimiento regular establecido. Se llevó a manejo quirúrgico, pero se evidenció un tumor irresecable y continuó en manejo oncológico con quimioterapia. Es el paciente con la mayor sobrevida global hasta el momento (92 meses).

Las funciones de supervivencia de Kaplan Meier con la supervivencia libre de 
enfermedad y global de la serie, se muestran en las gráficas 1 y 2 .

La mortalidad general fué del $15.8 \%$ (3 casos), sin embargo solo una de las muertes $(5,2 \%)$, fué por progresión de la enfermedad oncológica. Ese paciente presentó recaída local y regional a los 7 meses de terminar la neoadyuvancia. Fue llevado a cirugía, encontrando un tumor irresecable, por lo que requirió ostomia derivativa con quimioterapia paliativa. Presentó progresión y desenlace fatal a los 14 meses del manejo oncológico paliativo. Las otras 2 muertes fueron por causas diferentes a la enfermedad oncológica.

\section{Gráfica 1. Función de supervivencia libre de enfermedad}

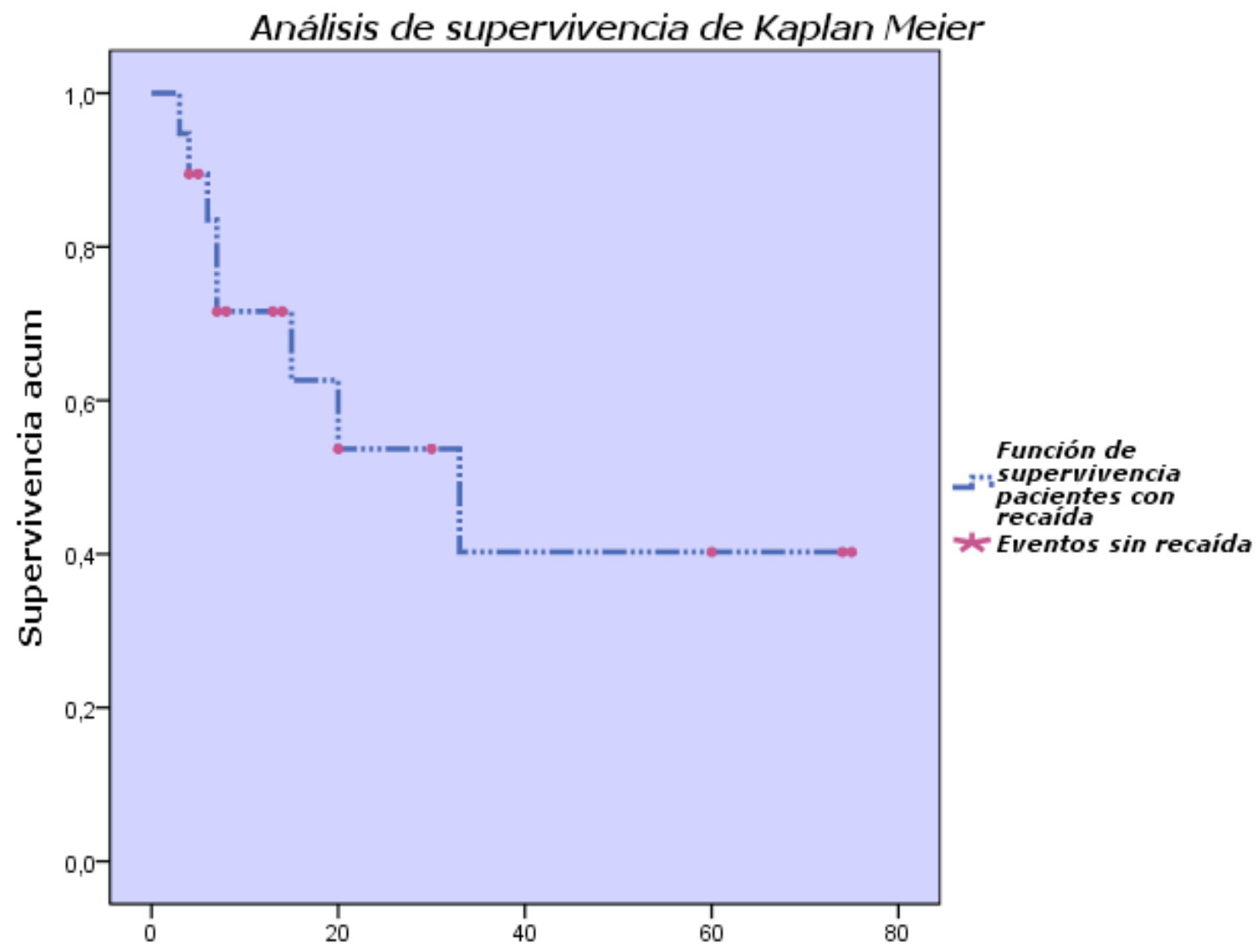

Tiempo de supervivencia libre de enfermedad 
Gráfica 2. Función de supervivencia global

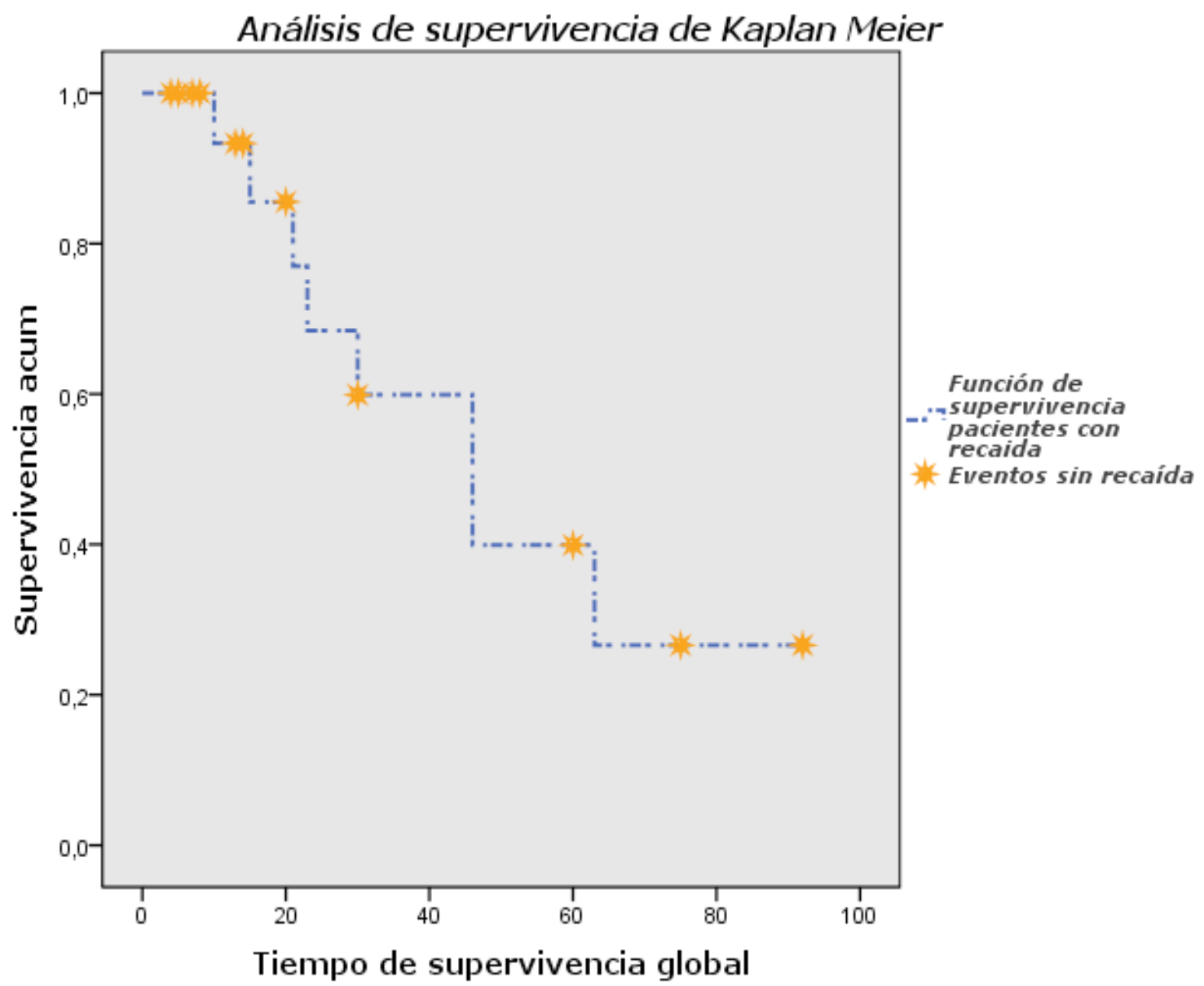




\section{DISCUSIÓN}

La Dra. Habr-Gama, fue la primera cirujano en determinar prospectivamente, el manejo no quirúrgico de pacientes con cáncer localmente avanzado de recto, después de determinarse respuesta clínica completa posterior a la neoadyuvancia ${ }^{19,23-24,35-39}$. Hay autores que han encontrado en sus series resultados similares y consideran viable la estrategia de observación, en casos seleccionados ${ }^{31-34,61}$. Aunque, el porcentaje reportado de respuesta clínica completa posterior a la neoadyuvancia es del 15 al 30 $\%{ }^{22}$, ese valor no fue posible de determinar en nuestra serie.

El Dr. Glynne-Jones y su grupo ${ }^{62}$, exponen que los resultados publicados por la Dra Habr-Gama, no son reproducibles sistemáticamente. Los puntos de discusión son: los protocolos de quimioterapia y radioterapia, las tasas de respuesta completa, el tiempo libre de recaída y los desenlaces después del manejo de la recaída, que no son claros en la mayoría de los artículos. Él expone, que sin claridad y reproducibilidad de los estudios, no es posible considerar la opción de seguimiento de manera regular, por que no es posible objetivizar los posibles desenlaces, para explicárselos al paciente y obtener la firma del consentimiento informado, que requiere cualquier alternativa de manejo, incluida la observación y seguimiento. Hughes y su grupo ${ }^{63}$, consideran que la alta tasa de recaída local temprana, sin una clara meseta de estabilización de la misma, significa que puede presentarse una recaída en cualquier momento del seguimiento, por lo que las muestras pequeñas de los estudios y la no estandarización de estos, hacen inviable la opción de seguimiento. En el Memorial Sloan Kettering Center de Nueva York ${ }^{64}$, se desarrolló un modelo analítico de decisión, para evaluar los beneficios de la cirugía radical vs el tratamiento conservador. Para ello, se tuvo en cuenta la capacidad de las herramientas diagnósticas actuales, para definir una 
respuesta clínica completa, la calidad de vida del paciente con o sin cirugía y el riesgo de recaída. La conclusión, es que la cirugía radical continúa siendo la mejor elección, para el promedio de pacientes con cáncer de recto y respuesta clínica completa postneoadyuvancia. El principal punto problema, es la limitación de los estudios diagnósticos actuales, para realizar una acertada y fiable reestadificación al terminar la quimiorradiación, que permita establecer criterios reproducibles de selección de pacientes, candidatos al manejo observacional.

Las nuevas técnicas por imagen (RNM dinámica o con perfusión y el PET-CT), aunque en estudio, se consideran de utilidad para la estadificación pre y postneoadyuvancia. Son capaces de detectar procesos celulares y fisiológicos, la perfusión local y actividad celular de zonas con posible infiltración tumoral y por ello se considera posible, que en el futuro permitan individualizar el manejo de cada paciente, al generar una evaluación adecuada y más precisa del compromiso tumoral ${ }^{30,65-67 .}$

Los reportes sobre el compromiso ganglionar son divergentes. Reportan tasas tan altas como del $5-10 \%$ para los ypT1 y del $20 \%$ para los ypT2 ${ }^{59}$ o menor del $2 \%$ para урт0/168. Todos los casos de cáncer de recto tratados con neoadyuvancia, pueden tener ganglios perirrectales. Un número importante de ellos son de $3 \mathrm{~mm}$ o menos y pueden ser positivos o negativos, para compromiso tumoral. Aunque los ganglios linfáticos metastásicos individuales presentan un diámetro mayor, el hallazgo único de estas adenopatías de 3mm, no descarta el compromiso regional, por lo que se recomienda actuar con precaución cuando se evidencien ${ }^{69}$.

La presencia de respuesta patológica completa, determinada en la pieza quirúrgica de una resección oncológica y el seguimiento de esos pacientes, ha demostrado prolongados tiempos de supervivencia libre de enfermedad y global, con una mínima tasa de recaída posterior. La importancia de esos estudios, es que nos llevan a considerar que hay pacientes con factores de buen pronóstico, que quizás puedan 
evitar el paso por el quirófano ${ }^{70-71}$.

Las biopsias de la cicatríz postneoadyuvancia, son de limitado valor clínico para descartar la presencia tumoral. Una biopsia negativa, no debe ser suficiente para evitar una resección quirúrgica, cuando sea necesaria ${ }^{72}$.

Cuando se identifican anormalidades residuales mínimas de la mucosa (menores de 3 cms, sin ulceración, sin estenosis), se ha considerado la realización de una resección local transanal. Si se confirma la invasión linfovascular, pobre diferenciación histológica, tamaño mayor de $3 \mathrm{cms}$ u otras características de mal pronóstico, es evidente que se presentan con mayor riesgo de compromiso ganglionar y por ende no serían candidatos al tratamiento quirúrgico local ${ }^{73}$. Aunque la respuesta clínica completa, determinada por la ausencia macroscópica de úlcera o lesión rectal tiene una alta especificidad, depender sólo de su hallazgo, en la selección de pacientes que van a ser manejados conservadoramente, se considera inapropiada. Deben existir otros valores de juicio, para descartar la realización del manejo quirúrgico radical ${ }^{73}$.

Hayden y su grupo ${ }^{74}$, encuentran que casi el $50 \%$ de los pacientes con tumor residual después de la neoadyuvancia, tienen celulas tumorales fuera de la úlcera visible y presentan enfermedad microscópica con mucosa normal. Esas células fueron identificadas, a más de $4 \mathrm{~cm}$ por fuera de los límites macroscópicos de la úlcera y se dirigian en cualquier dirección. El tejido cicatricial no presenta un patrón predecible y las celulas tumorales se evidencian en una disposición irregular, en relación con el centro de la úlcera visible. También encontro que esos pacientes con úlceras residuales, generalmente presentaban otras características de mal pronóstico, como invasión linfovascular. Estos resultados sugieren que el tratamiento con una resección local sería insuficiente y que el tradicional margen de $2 \mathrm{~cm}$ en dichas resecciones, es inadecuado ${ }^{74}$.

El antígeno carcinoembrionario se ha encontrado útil, en casos con respuesta clínica 
completa despues de neoadyuvancia, independientemente de otros factores clinicopatológicos. Aunque la sensibilidad en fumadores es equívoca ${ }^{39}$, se ha considerado como factor pronóstico de sobrevida en algunos estudios. También se ha sugerido, el aumento de intensidad de la quimioterapia adyuvante, cuando los valores de ACE preneoadyuvancia son marcadamente elevados ${ }^{40}$.

Aunque esten aún en investigación y no sea clara su significacia como factores pronósticos, la determinación de marcadores inmunohistoquímicos como p53, Ki67 y moleculares como Kras podrian correlacionarse con el comportamiento del tumor y la respuesta a los tratamientos sobre él ${ }^{42-50}$. En un reporte de Lin et $\mathrm{al}^{46}$, los autores comparan la expresión de p53, p27 y bcl-2 antes y después de la terapia neoadyuvante, mostrando un incremento de p27 y bcl-2 en los especímenes resecados, al compararse con la biopsia inicial, considerándose que la positividad de p27 parece mejorar el pronóstico. Estudios in vitro ${ }^{45}$, demuestran que un p53 normal es requerido en el tejido tumoral, para que responda adecuadamente a la quimioterapia basada en 5-FU. Su determinación antes de la neoadyuvancia, podría contribuir como predictor de respuesta a la quimioterapia ${ }^{45-46}$. Sin embargo, otros estudios hablan que el p53 es poco útil como buen predictor de respuesta a la quimioterapia y que para el bcl2 y ki67 no hay suficiente evidencia que avale su utilidad $^{47}$.

Aunque nuestra serie es pequeña, evidenciamos que 3 de 19 pacientes (15.8\%), cumplieron el seguimiento de 5 años, sin recaída. Las características biológicas de los tumores en los tres casos, no genera un patrón de referencia, para concluir que exista una relación causal de su expresión con el desenlace. Es de resaltar, que los tres pacientes presentaron regresión histológica completa postneoadyuvancia y las biopsias de la cicatriz fueron negativas. Los 8 pacientes que presentaron recaída antes de los 5 años, fueron llevados a manejo quirúrgico radical, determinándose irresecabilidad en 2 
de ellos. La supervivencia libre de enfermedad después del manejo quirúrgico, ha sido aceptable y sin alteraciones notables de la calidad de vida de los pacientes.

Recomendamos que sean presentados en junta multidisciplinaria, los pacientes con respuesta clínica completa y recaída local, sin establecerse compromiso regional o a distancia, con comorbilidades o rechazo al manejo quirúrgico radical, con la propuesta de realizar un manejo quirúrgico local, menos invasivo o endoscópico. El objetivo de esta estrategia y los riesgos que implica su implementación, deben ser ampliamente discutidos y entendidos por el paciente. Algunos grupos consideran el manejo local de la recaída, segun los conceptos de mal y buen pronóstico del cáncer superficial. Se consideran de buen pronóstico: pT1sm1, tamaño $<3 \mathrm{~cm}$, grado histológico 1 y 2 , ausencia de invasión linfovascular y predicción de resección Ro. El tratamiento propuesto, consiste en resección transanal de la lesión ${ }^{59}$. Otros grupos sugieren la realización de microcirugía transanal endoscópica, cuando hay factores de mal pronóstico, para evitar la realización de una resección radical quirúrgica. El grupo de la Dra Habr-Gama58, la plantea como opción en tumores residuales yT1-2 N0, que se estima, podrían presentar una tasa de recaída local del 15\%. La invasión linfovascular, se considera de mal pronóstico para la realización del procedimiento. Lo anterior ratifica la importancia de la selección e individualización de cada caso, aunque tampoco eso garantice la obtención de aceptables desenlaces ${ }^{58}$. El aún en curso, ensayo clínico randomizado fase 3 del Grupo Francés de investigación en la cirugía del cancer rectal, llamado GRECCAR-2 ${ }^{75}$, que compara la resección transanal con el manejo quirúrgico radical de tumores rectales $\mathrm{T} 2$ y $\mathrm{T} 3$, con buena respuesta local a la neoadyuvancia, nos brindará objetivos criterios de selección, para la implementación de una u otra estrategia de manejo.

La realización de un procedimiento quirúrgico radical, después de terminar la neoadyuvancia, entre la semanas 4 a 8 , permite el diagnóstico de solo $10 \%$ de los 
pacientes con respuesta clínica completa. La consolidación de la respuesta a la quimiorradiación, necesita tiempo y por eso debiera estadificarse después de la sexta semana de terminar el tratamiento y según la respuesta, considerar el manejo adicional $^{76-77}$. El ensayo clínico en curso, GRECCAR- $6^{78}$ (también del grupo Francés de investigación), busca determinar si la prolongación del tiempo de reevaluación después de la neoadyuvancia, esta asociado a mejores desenlaces quirúrgicos. En este estudio, aunque todos los pacientes vayan a ser llevados a cirugía, consideran prudente el esperar 7 a 11 semanas después de la quimiorradiación, para evaluar el tipo de respuesta a la neoadyuvancia. El objetivo del ensayo clínico, es determinar la respuesta patológica y evaluar los desenlaces en un seguimiento de 5 años. No contemplan la opción de manejo local u observacional en ningún caso.

El seguimiento actual de 5 años, para los pacientes con cáncer de recto tratados de manera estándar, se genera porque la mayor tasa de recurrencia ocurre alrededor de los primeros 3 años de realizado el manejo integral. Sin embargo, hay evidencia de que puede presentarse un nuevo tumor rectal (después de los 5 años de seguimiento), en los pacientes con respuesta clínica completa en seguimiento y observación, respecto al grupo regular de seguimiento. En las series de la Dra. Habr-Gama ${ }^{36-37}$, 3 de $13(23 \%)$ pacientes, tuvieron nuevamente tumor rectal después de los 5 años, por lo que sugiere un seguimiento mayor a ese tiempo. No hubo claridad de si se consideraba un segundo primario o una recaída (por definición, la recaída es reaparición del tumor dentro de los 5 años de seguimiento, posterior al manejo oncológico estándar). Hay que reportar de nuestra serie, que uno de los pacientes presentó un segundo primario rectal, con compromiso local y regional, a los 74 meses de seguimiento. Lo consideramos de esa manera y no una recaída, siendo fieles a las definiciones de nuestra metodología. El paciente tiene factores morfológicos y biológicos de buen pronóstico (no expresión de p53, no invasión linfovascular y ki67 bajo). Eso nos 
permite recalcar la importancia de extender el seguimiento al menos hasta los 10 años, mientras se continúan evaluando los desenlaces de este tipo de pacientes en otras series. Nuestra propuesta de manejo y seguimiento se expone en las gráficas 3 y 4.

Gráfica 3. Algoritmo de manejo del cáncer de recto

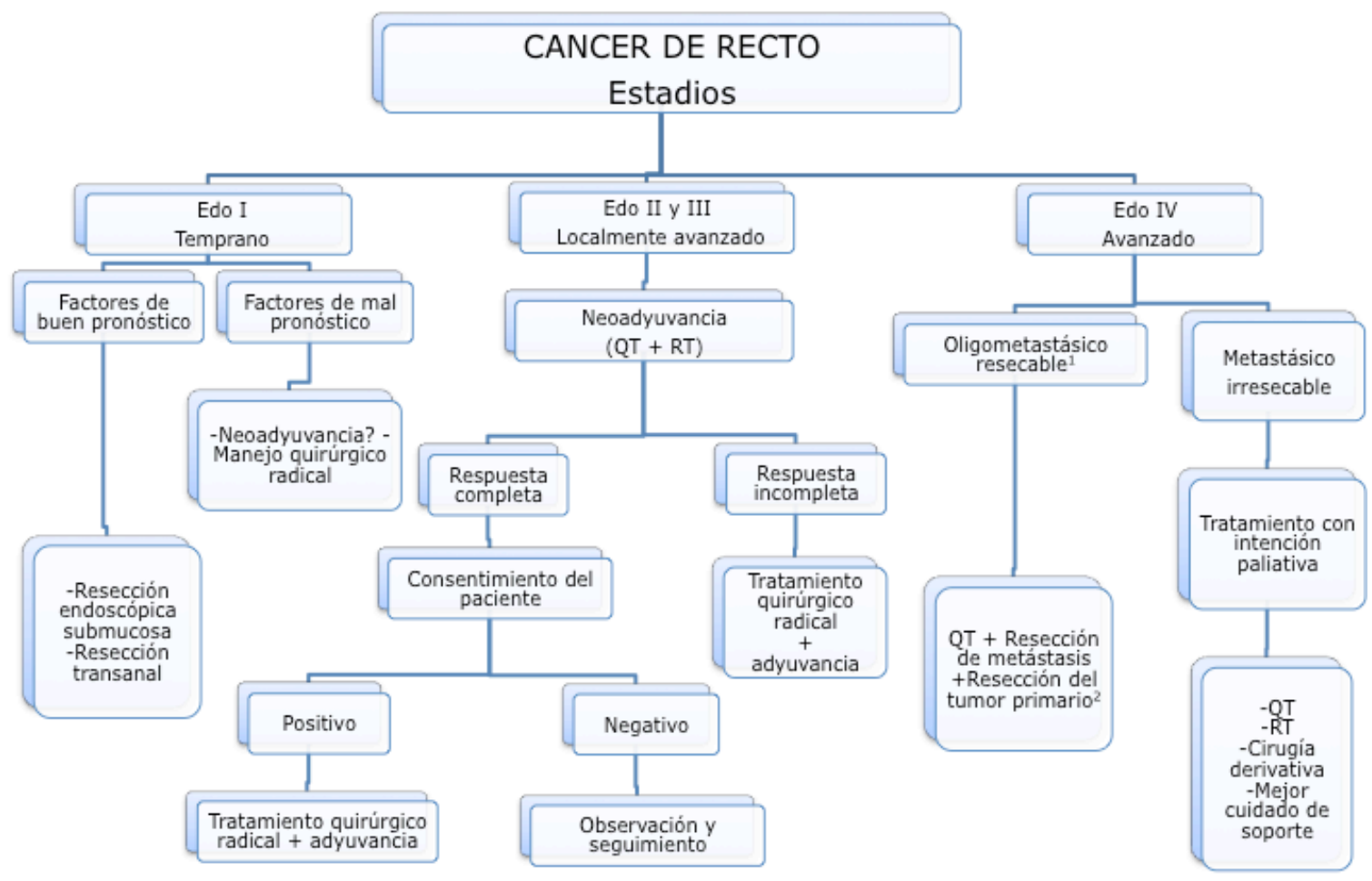

Edo: Estadio; QT: Quimioterapia; RT: Radioterapia

${ }^{1}$ Compromiso del tumor primario y metástasis exclusivas del higado, pulmón o peritoneal.

${ }^{2}$ El orden de los procedimientos es individualizado y depende del caso en particular.

El estudio de la expresión de perfiles genéticos en cáncer de recto y la caracterización de genes, directamente relacionados con la respuesta a la quimiorradiación, quizás permita en el futuro, la generación de un tratamiento personalizado ${ }^{79}$. 


\section{Gráfica 4. Seguimiento recomendado para los pacientes con respuesta clínica completa}

\section{post neoadyuvancia sin manejo quirúrgico radical}

-Primeros 3 años:

- Examen físico, marcadores tumorales, colonoscopia izquierda y biopsias de la cicatriz cada 3 meses

- $\quad$ RNM de pelvis + Tomografía tórax y abdomen cada 6 meses

- Colonoscopia total anual

-De los tres a 5 años:

- Examen físico, marcadores tumorales, colonoscopia izquierda y biopsias cada 6 meses

- $\quad$ RNM de pelvis + Tomografía tórax y abdomen + colonoscopia total anual

-De los 5 a 10 años:

- Examen físico + marcadores tumorales + colonoscopia total y biopsias + RNM de pelvis + Tomografía tórax y abdomen anual

Considerar PET-CT anual desde el primer año de seguimiento 


\section{CONCLUSIONES}

- La reestadificación postneoadyuvancia entre la semana 6 y 8, es adecuada para determinar el tipo de respuesta al tratamiento.

- Ratificamos el manejo quirúrgico radical posterior a la neoadyuvancia, en todos los pacientes con cáncer de recto, independientemente del tipo de respuesta clínica que presenten. Lo anterior porque no existe mayor respaldo bibliográfico, sobre la seguridad de un manejo no quirúrgico o mínimamente invasivo

- Los pacientes que no acepten ese manejo, y tengan respuesta clínica completa, deben evaluarse en junta multidisciplinaria, para determinar si son candidatos a la realización de una resección local y continuar en seguimiento estricto de su evolución.

- Aunque nuestra serie determinó una baja tasa de recaída local y regional, el tamaño de la muestra es muy pequeño, para recomendar la opción de seguimiento, a todos los pacientes con respuesta clínica completa, posterior a la quimiorradiación.

- La opción de seguimiento en nuestro medio, no es fácil, por lo que el manejo quirúrgico para nuestra población, nos parece la estrategia más responsable y segura.

- Ante la presencia de recaída local y/o regional, también ratificamos el manejo quirúrgico radical, con la misma consideración ya expuesta de realización de una resección local, por decisión del paciente.

- La muestra de nuestra serie y la ausencia de un patrón de relación entre las variables biológicas del tumor y los desenlaces, pone en evidencia, la necesidad de investigación suplementaria acerca del tema. 


\section{BIBLIOGRAFÍA}

1. Siegel R, Naishadham D, Jemal A. Cancer statistics, 2013. Ca Cancer J Clin 2013; 63:11-30.

2. V Van de Velde CJ, Boelens PG, Borras JM, et al. EURECCA colorectal: Multidisciplinary management: European consensus conference Colon \& Rectum. Eur J Cancer 2014; 50(1): 1.e1-1.e34.

3. Instituto Nacional de Cancerología E.S.E. Anuarios estadísticos 2003 - 2011.

4. Piñeros M, Pardo $C$, Gamboa $O$, et al. Atlas de mortalidad por cáncer en Colombia, tercera edición. Bogotá, 2010. Instituto Nacional de Cancerología E.S.E; Instituto Geográfico Agustín Codazzi. ISBN: 978-958-99697-2-4.

5. National Comprehensive Cancer Network. NCCN Clinical Practice Guidelines in Oncology, Rectal Cancer, Version 2.2014. URL: http://www.nccn.org/professionals/physician_gls/f_guidelines.asp.

6. Meredith $\mathrm{KL}$, Hoffe $\mathrm{SE}$, Shibata $\mathrm{D}$. The multidisciplinary management of rectal cancer. Surg Clin N Am 2009; 89: 177-215.

7. Akasu T, Linuma G, Takawa M, et al. Accuracy of high-resolution magnetic resonance imaging in preoperative staging of rectal cancer. Ann Surg Oncology 2009 Oct; $16(10): 2787-94$.

8. Cai $G, X u Y, Z$ hu J, et al. Diffusion-weighted magnetic resonance imaging for predicting the response of rectal cancer to neoadjuvant concurrent chemoradiation. World J Gastroenterol 2013; 19(33): 5520-5527.

9. Halefoglu AM, Yildirim $S$, Avlanmis $O$, et al. Endorectal ultrasonography versus phased array magnetic resonance imaging for preoperative staging of rectal cancer. World Journal Gastroenterology 2008; 14(22): 3504-3510. 
10.Bayraktar Y, Kav T. How useful is rectal endosonography in the staging of rectal cancer? World Journal of Gastroenterology 2010; 16(6): 691-697.

11. Chan $\mathrm{K}$, Welch $\mathrm{S}$, Walker-Dilks $\mathrm{C}$, et al. Evidence-based guideline recommendations on the use of Positron Emission Tomography imaging in Colorectal cancer. Clinical Oncology 2012; 24:232-249.

12. Glynne-Jones $R$, Kronfli $M$. Locally advanced rectal cancer: A comparison of management strategies. Drugs 2011; 71(9): 1153-1177.

13. Valentini V, Beets-Tan R, Borras JM, et al. Evidence and research in rectal cancer. Radiotherapy and Oncology 2008; 87:449-474.

14.McCarthy K, Pearson K, Fulton R, Hewitt J. Quimiorradiación preoperatoria para el cáncer rectal localmente avanzado no metastásico. Cochrane Database of Systematic Reviews 2012; Issue 12. Art. No: CD008368. DOI: 10.1002/14651858.CD008368.

15.Ceelen WP, Nieuwenhove YV, Fierens K. Preoperative chemoradiation versus radiation alone for stage II and III resectable rectal cancer. Cochrane Database Syst Rev 2009; Issue 1. Art No: CD006041. DOI: 10.1012/14651858.CD006041.pub2.

16. Conde S, Borrego $M$, Teixeira $T$, et al. Neoadjuvant oral vs. infusional chemoradiotherapy on locally advanced rectal cancer: Prognostic factors. Rep Pract Oncol Radiother 2013; 18(2): 67-75.

17.Sung UL, Dae YK, Sun YK, et al. Comparison of two preoperative chemoradiotherapy regimens for locally advanced Rectal cancer: Capecitabine alone versus capecitabine plus irinotecan. Radiation Oncology 2013; 8:258.

18.Viani GA, Stefano EJ, soares FV, et al. Evaluation of biologic effective dose and schedule of fractionation for preoperative radiotherapy for rectal cancer: Metaanalyses and meta-regression. Int. J. Radiation Oncology Biol Phys 2011; Vol. 80(4): 985-991. 
19. Habr-Gama A, M.D., Perez RO, Nadalin W. Long-term results of preoperative chemoradiation for distal rectal cancer correlation between final stage and survival. J Gastrointest Surg 2005; 9:90-101.

20.Rullier E, Denost Q, Vendrely V, et al. Low Rectal Cancer: Classification and Standardization of Surgery. Dis Colon Rectum 2013; 56: 560-567.

21.Evans J, Tait D, Swift I, et al. Timing of Surgery Following Preoperative Therapy in Rectal Cancer: The Need for a Prospective Randomized Trial? Dis Colon Rectum $2011 ; 54: 1251-1259$.

22. Weisser M, Beets-Tan $R$ and Beets $G$. Management of complete response after chemoradiation in rectal cancer. Surg Oncol Clin N Am 2014; 23 (1): 113-125.

23. Habr-Gama A, Perez RO, Wynn G, et al. Complete clinical response after neoadjuvant chemoradiation therapy for distal rectal cancer: Characterization of clinical and endoscopic findings for standardization. Dis Colon Rectum 2010; $53: 1692-1698$.

24.Habr-Gama A, Perez R, Proscurshim I, et al. Complete clinical response after neoadjuvant chemoradiation for distal rectal cancer. Surg Oncol Clin N Am 2010; 19:829-845.

25. Mignanelli ED, Campos-Lobato LF, Stocchi $L$, et al. Downstaging after chemoradiotherapy for locally advanced rectal cancer: Is there more (tumor) than meets the eye? Diseases of the Colon \& Rectum 2010; Vol 53: 3.

26. Thies S. and Langer R. Tumor regression grading of gastrointestinal carcinomas after neoadjuvant treatment. Front Oncol 2013; 3:262. Doi: 10.3389/fonc.2013.00262.

27.Franklin JM, Anderson EM, Gleeson FV. MRI features of the complete histopathological response of locally advanced rectal cancer to neoadjuvant chemoradiotherapy. Clinical Radiology 2012; 67: 546-552.

28. Mak $D$, Joon $D L$, Chao $M$, et al. The use of PET in assessing tumor response after 
neoadjuvant chemoradiation for rectal cancer. Radiotherapy and Oncology 2010; 97: 205-211.

29. Hongyoon C, Hai-jeon Y, Tae Sung K, et al. Voxel-based dual-time 18F-FDG parametric imaging for rectal cancer: differentiation of residual tumor from postchemoradiotherapy changes. Nuclear Medicine Communications 2013; 34:11661173.

30.Figueiras RG, Domínguez PC, Dorrego RG, et al. Prognostic factors and functional imaging in rectal cancer. Radiología 2012; 54(1): 45-58.

31.Dalton RSJ, Velineli $R$, Osborne $M E$, et al. $A$ single centre experience of chemoradiotherapy for rectal cancer: is there potential for nonoperative management? Colorectal Disease $2011 ; 14: 567-571$.

32. O'Neill BP, Brown G, Heald RJ. Non-operative treatment after neoadjuvant chemoradiotherapy for rectal cancer. Lancet Oncol 2007; 8: 625-633.

33. Fischkoff KN, Ruby JA, Guillem JG. Nonoperative approach to locally advanced rectal cancer after neoadjuvant combined modality therapy: Challenges and opportunities from a surgical perspective. Clinical Colorectal Cancer 2011; Vol 10(4): 291-297.

34. Higgins KA, Willett CG, Czito BG. Nonoperative management of rectal cancer: Current perspectives. Clinical Colorectal Cancer 2010; Vol 9(2): 83-88.

35. Habr-Gama A, de Souza PM, Ribeiro $U$, et al. Low rectal cancer: Impact of radiation and chemotherapy on surgical treatment. Dis Colon Rectum 1998; 41: 1087-96.

36. Habr-Gama A, Perez RO, São Julião GP, et al. Nonoperative approaches to rectal cancer: A critical evaluation. Semin Radiat Oncol 2011; 21:234-239.

37. Habr-Gama A, Perez RO, Nadalin W, et al. Operative Versus Nonoperative Treatment for Stage 0 Distal Rectal Cancer Following Chemoradiation Therapy. Long-term Results. Ann Surg 2004; 240: 711-718.

38. Habr-Gama A, Perez RO, Proscurshim I, et al. Patterns of failure and survival for 
nonoperative treatment of stage c0 distal rectal cancer following neoadjuvant chemoradiation therapy. J Gastrointest Surg 2006; 10:1319-1329.

39.Perez RO, Sao Juliao G, Habr-Gama A, et al. The role of Carcinoembriogenic antigen in predicting response and survival to neoadjuvant chemoradiotherapy for distal rectal cancer. Dis Colon Rectum 2009; 52: 1137-1143.

40.Chung MJ, Chung SM, Kim JY, et al. Prognostic Significance of Serum Carcinoembryonic Antigen Normalization on Survival in Rectal Cancer Treated with Preoperative Chemoradiation. Cancer Res Treat 2013; 45(3): 186-192.

41.Liebig C, Ayala G, Wilks G, et al. Perineural Invasion Is an Independent Predictor of Outcome in Colorectal Cancer. J Clin Oncol 2009; 27:5131-5137.

42. Díez M, Perez J, Martín A. Marcadores tumorales de valor pronóstico en adenocarcinomas de colon y recto. Gastroenterología integrada 2001; 2(4): 207221.

43.Garcia-Aguilar J, Chen Z, Smith David, et al. Identification of a biomarker profile associated with resistance to neoadjuvant chemoradiation therapy in rectal cancer. Ann Surg 2011; 254(3): 486-493.

44.Chen Z, Duldulao MP, Li W, et al. Molecular diagnosis of response to neoadjuvant chemoradiation therapy in patients with locally advanced rectal cancer. J Am Coll Surg $2011 ; 212: 1008-1017$.

45. Lopez-Crapez E, Bibeau F, Thezenas S, et al. p53 status and response to radiotherapy in rectal cancer: a prospective multilevel analysis. $\mathrm{Br}$ J Cancer 2005; $92: 2114-2121$.

46. Lin LC, Lee HH, Hwang WS, et al. p53 and p27 as predictors of clinical outcome for rectal-cancer patients receiving neo-adjuvant therapy. Surg Oncol 2006; 15: 211216.

47.Kuremsky JG, Tepper JE and McLeod HL. Biomarkers for response to neoadjuvant 
chemoradiation for rectal cancer. Int J Radiat Oncol Biol Phys 2009; 74:673-688.

48. Kato $S$, Iida $S$, Higuchi $T$, et al: PIK3CA mutation is predictive of poor survival in patients with colorectal cancer. Int J Cancer 2007; 121:1771-1778.

49. Nosho K, Kawasaki $T$, Ohnishi $M$, et al: PIK3CA mutation in colorectal cancer: Relationship with genetic and epigenetic alterations. Neoplasia 2008; 10: 534-541.

50.Imamura $Y$, Morikawa $T$, Liao X, et al. Specific Mutations in KRAS Codons 12 and 13, and Patient Prognosis in 1075 BRAF Wild-Type Colorectal Cancers. Clin Cancer Res $2012 ; 18: 4753-4763$.

51.Bengala C, Bettelli S, Bertolini F, et al: Epidermal growth factor receptor gene copy number, K-ras mutation and pathological response to preoperative cetuximab, 5-FU and radiation therapy in locally advanced rectal cancer. Ann Oncol 2009; 20:469474.

52.Zeestraten ECM, Kuppen PJK, Van de Velde $\mathrm{CJH}$, et al. Prediction in rectal cancer. Semin Radiat Oncol 2012; 22:175-183.

53. Moureau-Zabotto L, Farnault B, De Chaisemartin C, et al. Predictive factors of tumor response after neoadjuvant chemoradiation for locally advanced rectal cancer. Int J Radiation Oncology Biol Phys 2011; 80(2): 483-491.

54.Guedj N, Bretagnol F, Rautou PE. Predictors of tumor response after preoperative chemoradiotherapy for rectal adenocarcinomas. Human Pathology $2011 ; 42: 1702-$ 1709.

55.Pucciarelli S, De Paoli A, Guerrieri M, et al. Local Excision After Preoperative Chemoradiotherapy for Rectal Cancer: Results of a Multicenter Phase II Clinical Trial. Dis Colon Rectum 2013; 56: 1349-1356.

56.Kundel Y, Brenner R, Purim O, et al. Is Local Excision After Complete Pathological Response to Neoadjuvant Chemoradiation for Rectal Cancer an Acceptable 
Treatment Option? Dis Colon Rectum 2010; 53:1624-1631.

57.Bujko K, Sopyloy R, Kepka L. Local excision after radio (chemo) therapy for rectal cancer: is it safe? Clinical Oncology 2007; 19:693-700.

58.Perez RO, Habr-Gama A, Lynn PB, et al. Transanal Endoscopic Microsurgery for Residual Rectal Cancer (ypT0-2) Following Neoadjuvant Chemoradiation Therapy: Another Word of Caution. Dis Colon Rectum 2013; 56: 6-13.

59. Colombo PE, Patani N, Bibeau $F$, et al. Clinical impact of lymph node status in rectal cancer. Surgical Oncology 2011; 20: $227-233$.

60. Habr-Gama A, Perez RO, Sabbaga J, et al. Increasing the rates of complete response to neoadyuvant chemoradiotherapy for distal rectal cancer: Results of a prospective study using additional chemotherapy during the resting period. Diseases of the Colon \& Rectum 2009; 52(12): 1927-1934.

61.Lim L, Chao M, Shapiro J, et al. Long-Term Outcomes of Patients with Localized Rectal Cancer Treated with Chemoradiation or Radiotherapy Alone Because of Medical Inoperability or Patient Refusal. Dis Colon Rectum 2007; 50: 2032-2039.

62.Glynne-Jones $R$, Wallace $M$, Livingstone JIL, et al. Complete clinical response after preoperative chemoradiation in rectal cancer: Is a "wait and see" policy justified? Diseases of the Colon \& Rectum 2008; Vol 51: 10-20.

63. Hughes R, Harrison M, Glynne-Jones R. Could a wait and see policy be justified in T3/4 rectal cancers after chemo-radiotherapy? Acta Oncológica 2010; 49: 378-381.

64. Neuman HB, Elkin EB, JG Guillem, et al. Treatment for Patients with Rectal Cancer and a Clinical Complete Response to Neoadjuvant Therapy: A Decision Analysis. Dis Colon Rectum 2009; 52(5): 863-871.

65.Jacobson $O$ and Chen $X$. Interrogating Tumor Metabolism and Tumor Microenvironments Using Molecular Positron Emission Tomography Imaging. Theranostic Approaches to Improve Therapeutics. Pharmacol Rev 2013; 65:1214- 
1256.

66. Lambregts DM, Maas M, Bakers FC, et al. Long-term Follow-up Features on Rectal MRI During a Wait-and-See Approach After a Clinical Complete Response in Patients With Rectal Cancer Treated With Chemoradiotherapy. Dis Colon Rectum 2011; 54: $1521-1528$.

67. Haustermans K, Debucquoy A and Lambrecht M. The ESTRO Breur Lecture 2010: Towards a tailored patient approach in rectal cancer. Radiotherapy and Oncology $2011 ; 100: 15-21$.

68.Smith FM, Waldron D, Winter DC. Rectum-conserving surgery in the era of chemoradiotherapy. Br J Surg 2010; 97:1752-1764.

69.Perez RO, Pereira GV, Proscurshim I, et al. Lymph Node Size in Rectal Cancer Following Neoadjuvant Chemoradiation-Can We Rely on Radiologic Nodal Staging After Chemoradiation? Dis Colon Rectum 2009; 52: 1278-1284.

70. Martin ST, Heneghan MH, Winter DC. Systematic review and meta-analysis of outcomes following pathological complete response to neoadjuvant chemoradiotherapy for rectal cancer. British Journal of Surgery 2012; 99:918-928.

71. Maas $M$, Nelemans $P J$, Valentini $V$, et al. Long-term outcome in patients with a pathological complete response after chemoradiation for rectal cancer: a pooled analysis of individual patient data. Lancet Oncol 2010;11:835-44.

72. Perez RO, Habr-Gama A, Pereira GV, et al. Role of biopsies in patients with residual rectal cáncer following neoadjuvant chemoradiation after downsizing: can they rule out persisting cancer? Colorectal Disease $2011 ; 14: 714-720$.

73.Smith FM, Chang KH, Sheahan $\mathrm{K}$, et al. The surgical significance of residual mucosal abnormalities in rectal cancer following neoadjuvant chemoradiotherapy. British Journal of Surgery 2012; 99:993-1001.

74. Hayden DM, Jakate S, Mora MC, et al. Tumor Scatter After Neoadjuvant Therapy for 36 
Rectal Cancer: Are We Dealing with an Invisible Margin? Dis Colon Rectum 2012; 55: $1206-1212$.

75. Rullier $E$, Rouanet $\mathrm{P}$, Michot $\mathrm{F}$, et al. Local versus rectal excision in downstaged low rectal cancer after radiochemotherapy: preliminary results of the randomized GRECCAR 2 trial. (NCT00427375). Colorectal Disease J 2013; 15(s3): 4-12.

76. Nyasavajjala SM, Shaw AG, Khan AQ, et al. Neoadjuvant chemo-radiotherapy and rectal cancer: can the UK watch and wait with Brazil? Colorectal Disease 2010; 12: 33-36.

77.Perez RO, Habr-Gama A, Sao Juliao GP, et al. Optimal Timing for Assessment of Tumor Response to Neoadjuvant Chemoradiation in Patients With Rectal Cancer: Do All Patients Benefit From Waiting Longer Than 6w? Int J Rad Oncol Biol Phys 2012; 84(5): 1159-1165.

78. Lefevre JH, Rousseau A, Svrcek $M$, et al. A multicentric randomized controlled trial on the impact of lengthening the interval between neoadjuvant radiochemotherapy and surgery on complete pathological response in rectal cancer (GRECCAR-6 trial): rationale and design. BMC Cancer 2013, 13:417.

79.Palma $P$, Cuadros $M$, Conde-Muíño $R$, et al. Microarray Profiling of Mononuclear Peripheral Blood Cells Identifies Novel Candidate Genes Related to Chemoradiation Response in Rectal Cancer. PLoS One 2013; 8(9): e74034. 